\title{
Jewish Displaced Persons in Italia (1945-1950)
}

\section{Introduzione. Italia, Porta di Sion}

Grazie alla sua posizione strategica per la partenza di navi verso la Palestina, tra il 1945 e il 1948 transitarono e sostarono in Italia decine di migliaia di displaced persons (Dps) di religione ebraica'. La questione dei Jewish DPs ${ }^{3}$ deve essere contestualizzata nella più ampia «crisi dei profughi» che investiva l'Europa nel dopoguerra. All'indomani della fine del conflitto, il Vecchio Continente era profondamente mutato. In tutto il suo territorio avvenne una vera e propria tra-

Letteralmente "persone spostate", owvero profughi. Lo statuto di displacement veniva riconosciuto a coloro che erano privi di documenti e impossibilitati a prowedere al proprio sostentamento. Per un approfondito inquadramento storico e giuridico, si consulti: Salvatici 2008.

2 Si trattava di sopravvissuti ai lager, scampati alle deportazioni e partigiani, a cui si aggiunsero altri ebrei in fuga dai pogrom dell'Europa orientale scoppiati a partire dal 1946. Tra la fine del 1944 e l'estate del 1946 in Polonia si registra la seguente ondata di pogrom: Kiev (fine 1944); Rzeszòv (12 giugno 1945); Cracovia (11 agosto 1945); Sosnowiec (25 ottobre 1945); Lublino (19 novembre 1945), Kielce (4 luglio 1946). Un'altra ondata di pogrom scoppia in Romania tra la fine del 1946 e l'inizio del 1947. II caso di Kielce ebbe una notevole risonanza anche sulla coeva stampa ebraica italiana, per esempio il "Bollettino della Comunità ebraica di Milano" e "Israel". A scatenare un vero e proprio «esodo di ebrei dalla Polonia» che si riversavano in Italia attraverso l'Austria e a preoccupare la comunità ebraica internazionale erano state soprattutto le parole del cardinale Hlond, Primate cattolico della Polonia. Subito dopo i fatti di Kielce egli giustificò le violenze ai danni degli ebrei polacchi accusandoli di collaborazionismo coi comunisti. e Austria. 
sformazione demografica ${ }^{4}$ Judt 2007]. Non era la prima volta che profughi ebrei stranieri si trovano temporaneamente su territorio italiano come rifugiati. Fin dal 1933, infatti, l'Italia era stata terra d'approdo per numerosi ebrei in fuga dal nazismo che potevano ottenere facilmente un permesso di soggiorno in Italia per lavorares [Voigt 1996]. Già nell'autunno del '43 l'Italia si configura come terreno privilegiato di immigrazione ed emigrazione ebraica. Cominciano gli jugoslavi che scappano dall'arrivo dei soldati della Wehrmacht'; poi, dal 1945, i sopravvissuti ai lager che giungono tramite il valico di Tarvisio e il Passo del Brennero. A partire dall'estate del 1947 si aprì un ulteriore fronte: il Passo dei Tauri in Alto Adige ${ }^{8}$. Gli ebrei che giungono clandestinamente in Italia hanno come obiettivo l'Aliyah Beth', possibile per la posizione geografica strategica della penisola, per contingenze di politica interna ed estera [Enardu 1986; Toscano 1990; Romano 2000]; e per la capillare organizzazione del Mossad Le'Aliyah Beth ${ }^{10}$. Grazie a queste condizioni tra il 1944 e il 1948 l'Italia fu teatro della partenza clandestina di almeno 65 navi cariche di profughi ebrei diretti in Eretz Israel (Terra di Israele) [Sereni 1973; Toscano 1990].

La gestione dei Jewish DPs fu estremamente complessa. Soprattutto con l'obiettivo di evitare un enorme afflusso verso la Palestina, essi furono suddivisi nei campi profughi internazionali in base al loro paese d'origine, non tenendo conto della loro peculiarità come sopravvissuti ebrei. Questa scelta era causata dalla

Dal 1938-1939 fino al 1946 circa sessanta milioni di cittadini europei cambiarono residenza a causa dei progetti egemonici della Germania nazista, degli spostamenti coatti e delle persecuzioni razziali. Teniamo presente infatti che alcuni paesi vissero sei anni di conflitto (1939-1945) con effetti disastrosi; solo la Germania contò otto milioni di morti e I'Urss quasi venti.

L'approvazione delle leggi razziali chiarì definitivamente l'ambivalente atteggiamento del fascismo nei confronti degli ebrei. II decreto legge del 7 settembre 1938-XVI, n. 1381 colpiva innanzitutto gli ebrei stranieri, privandoli del diritto di soggiorno. Sulla genealogia delle Leggi Razziali italiane si veda in particolare: Sarfatti 2005.

6 I profughi i raggiungeranno le coste pugliesi, generalmente via mare e con imbarcazioni di fortuna. A Bari era attiva la Brigata partigiana jugoslava d'Oltremare, che ebbe anche un distaccamento ebraico fondato nell'autunno del 1943. Una volta organizzato ed addestrato, questo "plotone ebraico" - composto da esuli di varie nazionalità - partì da Monopoli per la Jugoslavia alla fine del 1944. Esso fu però decimato dalle truppe naziste e dalle terribili condizioni in cui i soldati si vennero a trovare.

Dove era stazionata la Jewish Infantry Brigade Group (la Brigata ebraica).

II Passo dei Tauri è tuttora conosciuto dalla popolazione locale come «passo degli ebrei». Per un'approfondita descrizione delle modalità d'arrivo e d'accoglienza dei Jewish displaced persons, si veda: Villani 2009; Villani 2010.

Nome in codice in ebraico per definire l'emigrazione nella Palestina sotto mandato britannico in violazione al libro bianco (1934-1948).

La branca dell'Haganah (dall'ebraico "La difesa", nome dell'organizzazione militare ebraica che opera in Palestina fino al 1948) che coordinava tutte le operazioni relative all'emigrazione ebraica in Palestina. 
spinosa «questione palestinese» e dal delicato contesto politico internazionale. Nel 1917 il ministro degli Esteri inglese Balfour aveva riconosciuto ufficialmente al movimento sionista il diritto alla creazione di uno Stato ebraico in Palestina, legittimando così l'arrivo, già dagli anni Venti, di massicci flussi migratori che causarono i primi scontri tra arabi e coloni ebrei. La questione palestinese divenne nodale dal 1945. La Gran Bretagna, che dal 1920 al 1947 governò la Palestina attraverso un mandato, provò a limitare l'immigrazione ebraica, causando forti tensioni con il movimento sionista e con la sua ala più estremista. Dal 1947 la Gran Bretagna ritirò le sue truppe e rimise il compito di decidere sulle sorti della Palestina all'Onu, che nel 1948 proclamò la nascita dello Stato di Israele, immediatamente respinta dagli arabi.

\section{Rinascita ebraica in Italia}

L'Aliyah Beth era solo culmine di un lungo processo che si svolgeva sul territorio italiano e che richiese uno sforzo organizzativo imponente". La maggior parte dei Jewish DPs furono assistiti dall'American Joint Distribuition Commitee (Joint) e da altri enti internazionali ebraici, in primis l'Organization for the Jewish refugees in Italy (Ojri), l'Organization for rehabilitation through training (Ort) e l'Oeuvre de secours aux enfants (Ose). Al momento del loro arrivo in Italia, superata un prima fase all'interno di strutture di prima accoglienza [Villani 2009; Villani 2010], essi furono trasferiti in strutture idonee ad una permanenza più duratura: campi profughi internazionali (organizzati dall'United Nations Relief and Rehabilitation Administration - Unrra e in seguito dalla International Refugee Organization Iro), kibbutz, hachsharah ${ }^{12}$, case stabili per bambini, colonie estive e colonie diurne. I primi campi profughi furono allestititi nel Sud Italia, in Puglia [Terzulli 1995; Leuzzi 2006], poi nel resto della penisola. Come emerge dagli studi e dalle testimonianze note fino ad ora [Pfanzelter 2002; Villa 2005; Pirani 2008; Kokkonen 2008; Vincon 2009; Villani 2010; Paganoni 2010; Ravagnan 2012] all'interno di queste strutture si svolgeva una vera e propria vita ebraica. Gli anni del transito

Proprio per questa ragione fin dal 1945 furono attivi in Italia Yehuda Arazi (1907-1958), membro dell'Haganah, e Ada Sereni. Quest'ultima, moglie di Enzo Sereni, scrittore e fervente sionista, emigrò in Palestina nel 1928 assieme a lui e fu tra i fondatori del grande kibbutz israeliano Givat Brenner. Nel 1944 raggiunse I'Italia per cercare suo marito, arruolatosi nella Brigata ebraica. Egli, arrestato dai nazisti dopo essersi fatto catapultare dalle forze alleate nel Nord della penisola, venne deportato a Dachau, dove morì il 18 novembre dello stesso anno. Ignara di cose fosse successo al marito, la Sereni sbarcò a Napoli il 7 luglio del 1945 per mettersi in contatto con l'organizzazione clandestina dell'Aliyah Beth. Cfr. Marzano, 2003. 
furono di formazione per i sopravvissuti che si definivano "She'ertih Hapleitah»" [Mankowitz 2002, Ravagnan 2012]. La loro vita era sospesa tra l'elaborazione dei lutti e la necessità di ricostruire un presente e un futuro. Quando si troverà a Katowice, nel suo memorabile viaggio di ritorno verso casa narrato ne La tregua, un avvocato polacco dirà a Primo Levi «la guerre n'est pas finie» [Levi, 1963], affermazione destinata a diventare celebre il cui significato è racchiuso nel titolo stesso dell'opera di Levi: una tregua appunto. Per migliaia di persone la liberazione dai lager non rappresentò la pace, ma solo la sospensione degli orrori; e il processo di piena riacquisizione dei diritti fu lungo e complesso ${ }^{14}$. Nei campi profughi da parte degli enti ebraici vennero organizzate scuole e asili, corsi professionali, momenti ricreativi legati all'arte, al teatro e allo sport. Da parte del Joint un'attenzione primaria venne data alla formazione dei bambini, orfani o nati dalle nuove unioni celebrate in Italia tra sopravvissuti [Menici 2003]. Per loro vennero organizzati momenti ad hoc e specifiche strutture ${ }^{15}$. I matrimoni celebrati nei campi profughi furono diverse centinaia in tutta Italia e diventarono il simbolo più evidente della volontà di riscattarsi dalla solitudine a cui la Shoah aveva condannato questi giovani, e della loro voglia di guardare al futuro' ${ }^{16}$.

\section{I profughi ebrei stranieri in Emilia Romagna}

Nel panorama nazionale precedentemente illustrato, l'Emilia Romagna rappresenta un case study di particolare interesse e fecondo di riflessioni a livello storico, come si cercherà di mettere in luce nel presente contributo ${ }^{17}$. Tra il 1945 e il 1948 in questa regione, ma con particolare intensità nelle province di Modena $\mathrm{e}$ Reggio Emilia, vengono allestiti numerosi campi profughi, diversi per tipologia,

\footnotetext{
È una formula biblica in ebraico che può essere tradotta con «il rimanente che è stato salvato»; oppure, veicolando un'immagine attiva del sopravvissuto, con «il rimanente salvifico».

Anche per gli ebrei italiani la guerra non fini: il complesso processo di abrogazione delle leggi razziali, il trauma del ritorno a casa, le ricerche dei dispersi, la ricerca del vecchio posto di lavoro, il recupero dei beni confiscati. Cfr. Sarfatti 1998; Schwarz 2004.

La più famosa fu la casa stabile per bambini di Selvino allestita nell'ex colonia fascista nelle montagne bergamasche $e$ organizzata sotto l'egida del combattente Moshe Ze'iri, attualmente al centro di un interessante dibattito e di un'ipotesi di riqualificazione attraverso la costruzione di un museo/centro polivalente.
}

Per quanto riguarda i matrimoni tra ebrei nei campi profughi e più in generale la dimensione familiare e di genere, si veda: Königseder e Wetzel 2001; Grossmann 2002; Grossmann 2007; Myers Feinstein 2009.

In questa sede si offrono, infatti, alcuni dati preliminari sui profughi stranieri in Emilia Romagna, risultanti dallo spoglio dei documenti della comunità ebraica di Modena ed altri archivi locali attuato per la ricerca di dottorato "I campi profughi per jewish displaced persons tra storia, ricostruzione e memoria", attualmente in corso presso presso l'Università di Trieste. 
funzione e gestione. La situazione che emerge è estremamente frammentaria e complessa da delineare. Una congerie di fattori influisce sulla quotidianità dei profughi e sui loro rapporti con l'ambiente circostante: la tipologia della struttura, le condizioni materiali, l'isolamento o meno rispetto al centro urbano, la densità abitativa o la convivenza di displaced persons di diverse nazionalità e con differenti backgrounds. Consideriamo quanto potesse differire la permanenza in un kibbutz, dove c'era un'omogeneità sociale, culturale e religiosa da quella in un campo internazionale Unrra.

Un caso ancora più paradossale è il riutilizzo dell'ex campo d'internamento di Fossoli per la sistemazione anche di profughi ebrei, generalmente arrestati dalla polizia italiana perché non in possesso dei documenti di riconoscimento ${ }^{18}$. La notizia della riapertura di Fossoli si diffuse nell'aprile del 1946, contestualmente alla notizia della fuga di un quattordicenne ebreo dal campo [Villa 2005, 248]. I profughi ebrei, trattati alla stregua di delinquenti, erano costretti a convivere con criminali e prigionieri di guerra in condizioni di promiscuità, di precaria igiene e con poco cibo. La situazione peggiorò notevolmente nell'aprile del 1946, quando essi intentarono uno sciopero della fame per protestare contro le condizioni di vita all'interno del campo. Presso l'archivio della Comunità ebraica di Modena sono custodite le lettere scritte da alcuni di questi profughi e inviate al presidente della Comunità di Modena Gino Friedmann perché intercedesse a favore di un loro allontanamento da Fossoli ${ }^{19}$, trasferimento che non venne mai ottenuto se non dopo la chiusura del campo. Anche gli ebrei del campo profughi sito all'interno dell'ex caserma Zucchi di Reggio Emilia vivevano in una situazione piuttosto conflittuale a causa della convivenza nella stessa struttura di decine di nazionalità differenti ${ }^{20}$. L'acme venne raggiunto il $1^{\circ}$ maggio del 1946, quando essi furono vittime di un vero e proprio attacco organizzato da parte di profughi ucraini, prigionieri di guerra che avevano combattuto al fianco dei nazisti sotto il comando del generale Vlassov. A causa delle violenze ordite ai danni dei giovani militanti ebrei del campo, colpevoli secondo gli ucraini di aver esposto bandiere rosse e simulacri della Russia sovietica, perse la vita il giovane ebreo Israel [Villa 2005; 
Kokkonen 2011; Ravagnan 2012] $]^{21}$.

Decisamente diversa era la situazione presso il kibbutz Terrachini, situato in via Fratelli Cervi a Reggio Emilia, e presso quello modenese, allestito all'interno della villa proprietà del non ebreo dott. Bisbini ${ }^{22}$ a Fossalta di Modena, e presso l' $h a-$

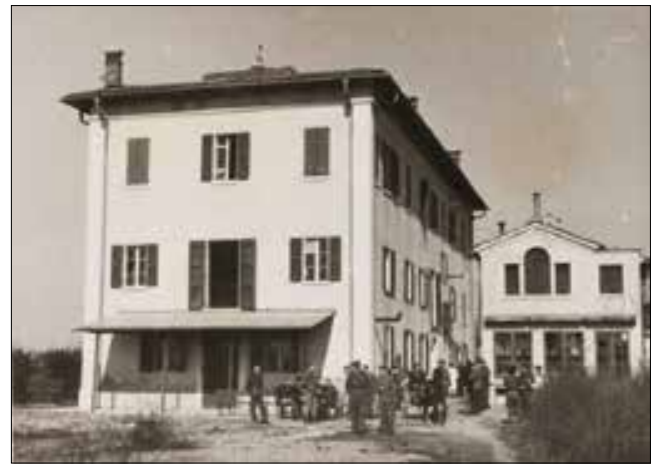

Fossalta, Villa Bisbini, 1946 [Biblioteca civica d'arte Luigi Poletti - Fondo Tonini, pos 1653]

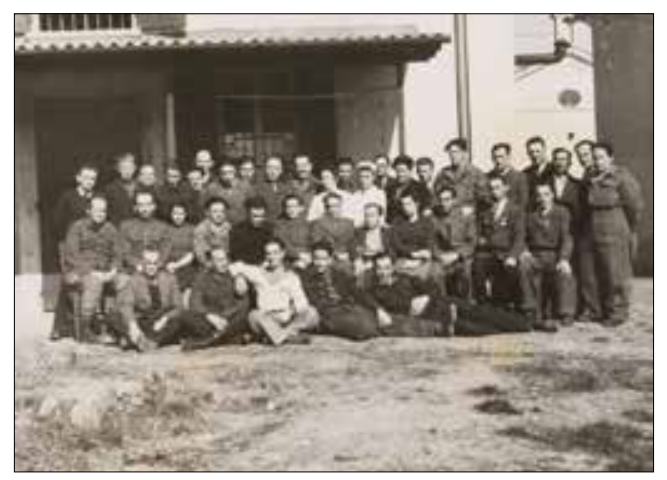

Fossalta, Villa Bisbini, 1946 [Biblioteca civica d'arte Luigi Poletti - Fondo Tonini, pos 1654] chsharah di Villa Emma a Nonantola in cui vivevano esclusivamente ebrei che studiavano e si formavano in vista dell'aliyah.

Si trattava, infatti, di luoghi piuttosto distanti rispetto ai centri abitati e questo contribuiva all'isolamento dei Jewish DPs rispetto alla popolazione italiana. In particolare, la vicenda di Villa Emma si presenta di grande interesse. Questo luogo, come noto ormai da diversi anni, a partire dal 1942 fu rifugio sicuro per una settantina di bambini e ragazzi ebrei provenienti dall'est Europa [Voigt 2002]. Grazie alla Delegazione per l'assistenza degli emigranti ebrei (Delasem), che pagò l'affitto della villa, essi poterono vivere e studiare in relativa tranquillità fino all' 8 settembre 1943. Poche ore dopo la notizia dell'armistizio don Arrigo Beccari e Giuseppe Moreali, grazie alla collaborazione e al silenzio della popolazione locale, riuscirono a nascondere questi ragazzi in attesa della loro fuga in Svizzera, che venne organizzata poche settimane dopo. A partire dal 1946 il medesimo luogo venne utilizzato per accogliere giovanissimi Jewish DPs, continuando così a scrivere un'importante storia di salvataggio e accoglienza da parte

Cfr. I dolorosi episodi di Reggio Emilia, "Israel", 35, 16 maggio 1946, 2; Cruento conflitto a Reggio tra profughi ebrei e russi bianchi, "Giornale dell'Emilia”, 4 maggio 1946. Per un resoconto dell'episodio si veda anche il protocollo firmato dai rappresentanti di tutti i movimenti chaluzistici del campo di Reggio Emilia il 6 maggio 1946. 
della comunità di Nonantola.

Di grande interessante appare anche il ruolo avuto nella gestione degli ebrei stranieri tra il 1945 e il 1948 dalla Comunità ebraica di Modena e dal suo presidente Gino Friedmann (1876-1964). Egli, che ebbe un ruolo di rilievo anche nella salvezza dei ragazzi di villa Emma come delegato modenese della Delasem, diede aiuto logistico, mate-

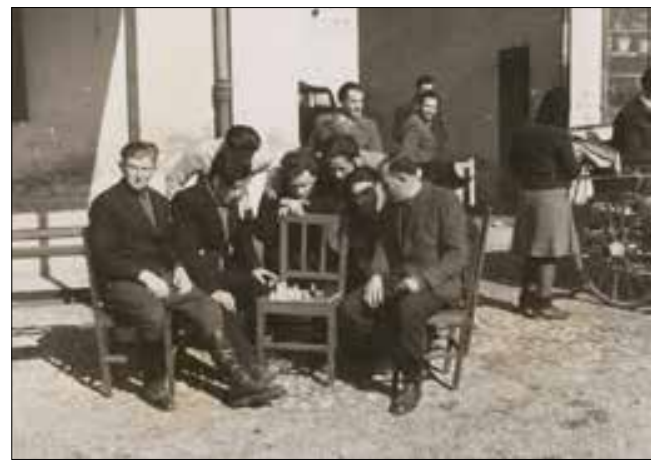

Fossalta, Villa Bisbini, 1946 [Biblioteca civica d'arte Luigi Poletti - Fondo Tonini, pos 1655] riale e burocratico agli ebrei stranieri. In particolare gli aiuti economici riguardavano le spese per il culto (per esempio per le circoncisioni rituali o la cucina kosher), le celebrazioni dei matrimoni tra profughi stranieri, le procedure burocratiche per l'emigrazione in Palestina e la contrattazione per gli affitti di Villa Bisbini e Villa Emma ${ }^{23}$.

\section{Ulteriori prospettive di ricerca}

Se sulle vicende relative all'emigrazione ebraica in Palestina tra il 1945 e il 1948 e sulle sue implicazioni rispetto alle politiche nazionali, sulle modalità di arrivo in Italia delle Jewish DPs e sull'organizzazione interna ai DP camps esistono ormai diversi studi, l'intento di questo contributo è stato di realizzare un focus che restituisse invece un segmento di storia praticamente sconosciuto alla storiografia e alla memoria locale. Sebbene quanto emerso fino ad ora ci restituisca un quadro piuttosto frammentato dei Jewish DPs, esso ci fa riflettere sulla complessità delle fasi di transizione e di ricostruzione. E' singolare che un pezzo di storia italiana del dopoguerra così gravido di conseguenze sia caduto per anni nell'oblio. Ignorate dalla storia nazionale italiana per lungo tempo, le vicende dei profughi ebrei stranieri in Italia nel dopoguerra sono infatti divenute oggetto di studio e attenzione solo di recente. Sarà, però, necessario nei prossimi anni perseguire un lavoro di ricerca approfondito per ricostruire questi anni e ricollocarli all'interno della storia nazionale italiana. Solo lo spoglio degli archivi comunali, dei fondi pubblici e privati, la catalogazione delle testimonianze dirette o indirette, a partire 
dalle seconde generazioni e della stampa locale, potrà infatti davvero restituirci il quadro completo di queste vicende.

\section{Bibliografia}

Bauer Y. 1998, Jewish Survivors in DP Camps and She'erith Hapletah, in Marrus M.R. (ed.) 1989, The Nazi Holocaust. Historical Articles on the Destruction of European Jews, vol. 9 The End of the Holocaust, Wesport-London: Meckler, 526-538

Capogreco C.S.1987, Ferramonti. La vita e gli uomini del più grande campo d'internamento fascista, 1940-1945, Firenze: Giuntina

Dinnerstein L. 1982, America and the Survivors of the Holocaust, New York: Columbia University Press

Di Sante C. (ed.) 2008, Il campo per gli “indesiderabili”. Documenti e immagini del "centro raccolta profughi stranieri” di Fossoli (1945-1947), Torino: Ega Editore

Enardu M. G. 1986, L’immigrazione illegale ebraica verso la Palestina e la politica estera italiana, 1945-1948, in "Storia delle relazioni internazionali", 1986, 147-164

Ferrara A. e Pianciola N. 2012, L'età delle migrazioni forzate, Esodi e deportazioni in Europa 1853-1953, Bologna: il Mulino

Gatrell P. 2013, The Making of the Modern Refugee, New York: Oxford University press

Grossmann A. 2002, Victims, Villains, and Survivors: Gendered Perceptions and SelfPerceptions of Jewish Displaced Persons in Occupied Postwar Germany, in "Journal of the History of Sexuality", 1-2

Grossmann A. 2007, Jews, Germans, and Allies. Close Encounters in Occupied Germany, Princeton and Oxford: Princeton University Press

Jockusch L. 2012, Collect and Record! Jewish Holocaust Documentation in Early Postwar Europe, New York: Oxford University Press

Judt J. 2007, Dopoguerra. Come è cambiata l'Europa dal 1945 ad oggi, Milano: Mondadori

Kokkonen S. 2008, Jewish Displaced Persons in Postwar Italy, 1945-1951, in "Jewish Political Studies Review", 1-2

Königseder A. e Wetzel J. 2001, Waiting for hope. Jewish displaced persons in postWorld War II Germany, Evanston: Northwestern University Press

Kulischer E.M.1948, Europe on the Move. War and population changes, 1917-47, New York: Columbia University Press

Lavsky H. 2002, New Beginnings. Holocaust Survivors in Bergen Belsen and the British Zone in Germany, 1945-1950, Detroit: Wayne State University Press

Lelli F. (ed.) 2004, Un'odissea dei nostri giorni, Lavello (PZ): Congedo Editore

Levi P. 1997, La tregua, Torino: Einaudi 
Leuzzi V.A. e Esposito G. (eds.) 2006, La Puglia dell'accoglienza. Profughi, rifugiati e rimpatriati nel Novecento, Bari: Irrsae Puglia-Istituto pugliese per la storia dell'antifascismo e dell'Italia contemporanea-Progedit

Mankowitz Z.W. 2002, Life between Memory and Hope. The Survivors of the Holocaust in Occupied Germany, Cambridge: Cambridge University Press,

Marrus M.R. 1985, The Unwanted: European Refugees in the Twentieth Century, New York: Oxford University Press

Marzano A. 2003, Una terra per rinascere, Gli ebrei italiani e l'emigrazione in Palestina prima della Guerra (1920-1940), Milano: Marietti

Menici S. 2003, L'opera del Joint in Italia. Un “Piano Marshall” ebraico per la ricostruzione, in «La Rassegna Mensile di Israele», 2, tomo 2, 593-617

Morpurgo G. 2008, Il violino liberato, Milano: Mursia

Myers Feinstein M. 2009, Holocaust Survivors in Postwar Germany, 1945-1957, Cambridge: Cambridge University Press

Paganoni M. (ed.) 2010, Per ricostruire e ricostruirsi, Astorre Mayer e la rinascita ebraica tra Italia e Israele, Milano: Franco Angeli

Patt A.J. e Berkowitz M. 2010, We are here. New approaches of Jewish Displaced Persons in Postwar Germany, Detroit: Wayne State University Press

Pirani S. 2008, Storia dell'Hakhsharah di Fano dal 1945 al 1948 attraverso i documenti e le interviste ai testimoni, Bologna: Patron [London 1956]

Ravagnan M. 2012, I campi Displaced persons per profughi stranieri in Italia (19451950), in «Storia e futuro, rivista di storia e storiografia», 30

Romano G. 2000, Gli indesiderabili. L'Italia e l'immigrazione clandestina ebraica in Palestina 1945-1948, in "Nuova storia contemporanea", 6, 2000

Rosen A. 2010, The Wonder of Their Voices. The 1946 Holocaust Interviews of David Boder, New York: Oxford University Press

Salvatici S. 2008, Senza casa e senza paese, profughi europei nel secondo dopoguerra, Bologna: il Mulino

Sarfatti M. 1998, Il ritorno alla vita, Firenze: Giuntina

Schwarz G. 2004, Ritrovare se stessi. Gli ebrei nell'Italia postfascista, Roma-Bari: Laterza

Sereni A. 1973, I clandestini del mare, l'emigrazione ebraica in terra d'Israele dal 1945 al 1948, Milano: Mursia

Terzulli F. 1995, Una stella tra i trulli. Gli ebrei in Puglia (1939-49), Bari: M. Adda,

Toscano M. 1990, La porta di Sion, l'Italia e l'immigrazione clandestina ebraica in Palestina (1945-1946), Bologna: il Mulino

Villa A. 2005, Dai lager alla terra promessa. La difficile reintegrazione nella «nuova Italia» e l'immigrazione verso il Medio Oriente (1945-1948), Milano: Guerini e associati 
Villani C. 2009, Milano, via Unione 5. Un centro di accoglienza per displaced persons ebree nel secondo dopoguerra, in «Studi Storici», 2, 333-370

Villani C. 2010, Infrangere le frontiere. L'arrivo delle displaced persons ebree in Italia (1945-1948): flussi, vie d'ingresso e politiche d'accoglienza, tesi di dottorato, Università di Trento

Vinçon S. 2009, Vite in transito. Gli ebrei nel campo profughi di Grugliasco (1945-1949), Torino: Zamorani

Voigt K. 1993, Il rifugio precario. Gli esuli in Italia dal 1933 al 1945, 2 voll., Firenze: La Nuova Italia

Voigt K. 2002, Villa Emma. Ragazzi ebrei in fuga. 1940 - 1945, Firenze:La Nuova Italia

\section{Risorse}

Displaced Persons Camps in Italy http://www.dpcamps.org/italy.html

Profughi ebrei in Puglia http://www.profughiebreinpuglia.it

University of Southern California Shoah Foundation http://college.usc.edu/vhi/

United State Holocaust Memorial Museum https://www.ushmm.org/

DP Camps and Hachsharot in Italy after the war Yad Vashem. The World Holocaust Remembrance Center http://yadvashem.org/yv/en/exhibitions/dp_camps_italy/index.asp 